\title{
Article \\ Semantic Reasoning of Product Biologically Inspired Design Based on BERT
}

\author{
Ze Bian ${ }^{1}$, Shijian Luo ${ }^{1,2, *}$, Fei Zheng ${ }^{3}$, Liuyu Wang ${ }^{4}$ and Ping Shan ${ }^{1}$ \\ 1 Department of Industrial Design, Zhejiang University, Hangzhou 310027, China; 11421074@zju.edu.cn (Z.B.); \\ 11621071@zju.edu.cn (P.S.) \\ 2 Ningbo Research Institute, Zhejiang University, Ningbo 315100, China \\ 3 Institute of Artificial Intelligence, College of Computer Science and Technology, Zhejiang University, \\ Hangzhou 310027, China; zfscgy2@zju.edu.cn \\ 4 Zhejiang Computer Federation, Hangzhou 310027, China; zjcia@zju.edu.cn \\ * Correspondence: sjluo@zju.edu.cn
}

Citation: Bian, Z.; Luo, S.; Zheng, F.; Wang, L.; Shan, P. Semantic Reasoning of Product Biologically Inspired Design Based on BERT. Appl. Sci. 2021, 11, 12082. https://doi.org/ 10.3390/app112412082

Academic Editor: Juan-Carlos Cano

Received: 22 November 2021

Accepted: 16 December 2021

Published: 18 December 2021

Publisher's Note: MDPI stays neutral with regard to jurisdictional claims in published maps and institutional affiliations.

Copyright: () 2021 by the authors. Licensee MDPI, Basel, Switzerland. This article is an open access article distributed under the terms and conditions of the Creative Commons Attribution (CC BY) license (https:// creativecommons.org/licenses/by/ $4.0 /)$.

\begin{abstract}
Bionic reasoning is a significant process in product biologically inspired design (BID), in which designers search for creatures and products that are matched for design. Several studies have tried to assist designers in bionic reasoning, but there are still limits. Designers' bionic reasoning thinking in product BID is vague, and there is a lack of fuzzy semantic search methods at the sentence level. This study tries to assist designers' bionic semantic reasoning in product BID. First, experiments were conducted to determine the designer's bionic reasoning thinking in top-down and bottom-up processes. Bionic mapping relationships, including affective perception, form, function, material, and environment, were obtained. Second, the bidirectional encoder representations from transformers (BERT) pretraining model was used to calculate the semantic similarity of product description sentences and biological sentences so that designers could choose the high-ranked results to finish bionic reasoning. Finally, we used a product BID example to show the bionic semantic reasoning process and verify the feasibility of the method.
\end{abstract}

Keywords: biologically inspired design; bionic reasoning; semantic matching

\section{Introduction}

\subsection{Product Biologically Inspired Design}

People get inspiration from one domain and apply it to another domain, which is called analogy [1]. Biologically inspired design (BID) takes biology as the source domain, and engineering, architecture, and industrial products can be target domains [2,3]. Product BID is a biologically inspired design in which the target domain is the product appearance.

The BID strategy can be divided into a top-down approach and a bottom-up approach. In the top-down approach [4], there is always a well-defined problem, and designers search for analogous solutions in nature to solve it. In the bottom-up approach, designers start from biology to find suitable design objects.

There are two significant processes in product BID: bionic reasoning and bionic expression (Figure 1). In bionic reasoning, designers search for creatures and products that are matched according to the design goal $[5,6]$. In bionic expressions, designers use the creature and product obtained by reasoning to draw detailed sketches and build 3D models accomplishing the product BID [7]. 


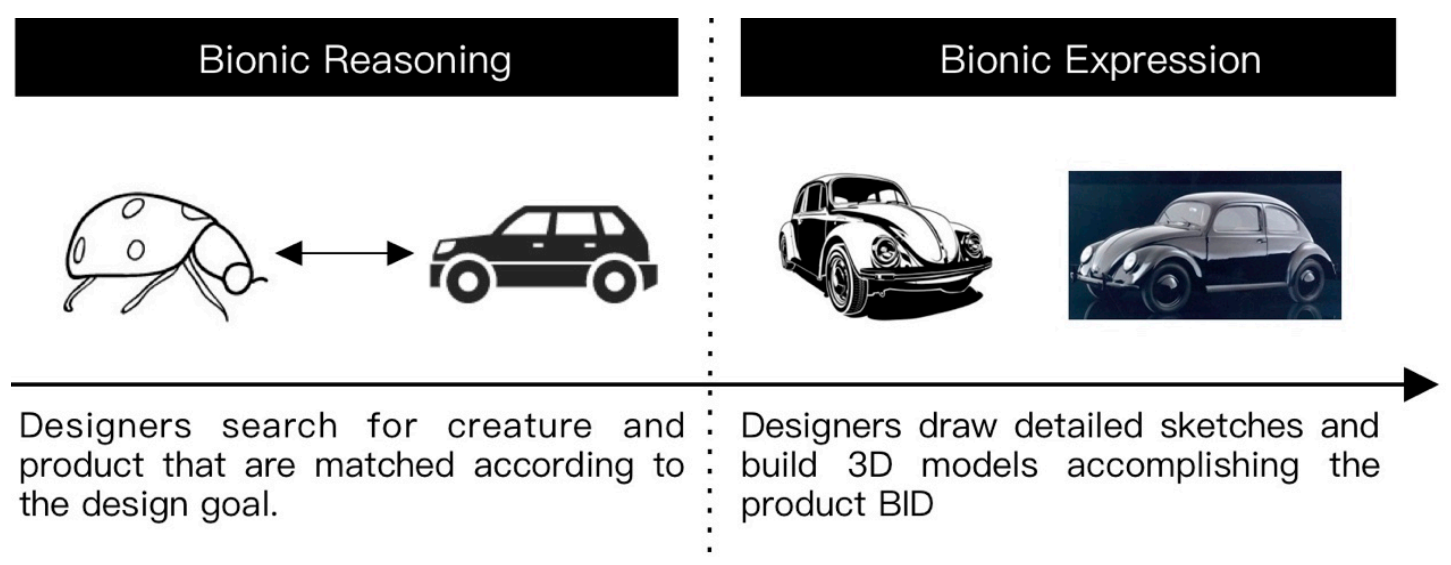

Figure 1. Product design process.

\subsection{Bionic Reasoning}

Bionic reasoning is important for product BID. Choosing related creatures and products can make designers' thinking and sketching in the subsequent bionic expression stage smoother, and the bionic product can convey harmonious imagery and aesthetics for users. This stage requires comprehensive knowledge of products, creatures and product design, which is difficult for designers, especially novices.

Several studies have attempted to assist designers in bionic reasoning accurately and efficiently through scientific methods, including (1) templates, (2) keyword-based search, (3) perceptual matching and (4) morphological matching.

(1) Templates are used to guide analogy thinking by asking designers to write down the corresponding information of the source domain and target domain and are proven to improve the accuracy of bionic reasoning $[8,9]$.

(2) Biology and engineering databases are established to accomplish bionic reasoning by keyword-based search, which is a precise search, and the results are limited [10]. To improve keyword-based search, a 'bridging' method is introduced to identify nonobvious but highly relevant keywords, including nouns and verbs [11-13]. Biological or engineering keywords are expanded by adding their troponym or hypernym from the online lexical WordNet.

(3) The perceptual matching method is another semantic approach for bionic reasoning. By scoring the affective perception of creatures and products with the same index, researchers obtain creatures and products that have similar affective perceptions for subjects. Luo et al. [14] and Yuan et al. [15] used perceptual evaluation to predict users' preference for creatures in BID.

(4) For morphological reasoning, Luo et al. [16] introduced a shape context algorithm to find a biological contour that is most similar to the given product contour. Zhu [17] used topological similarity to perform bionic morphological reasoning by analyzing the topological relation of organisms and products.

However, there are also limits in the above bionic reasoning methods. First, there is a lack of in-depth research on designers' ideation in bionic reasoning. In addition, 'bridging' expanded the search result, but it is still a precise search and can only search for words, not sentences. There is a lack of fuzzy semantic search methods at the sentence level. Finally, perception matching requires a large number of users to score, which is inconvenient and inefficient.

\subsection{Bidirectional Encoder Representations from Transformers (BERT) Pretraining Model}

BERT is a pretraining language model proposed by Google that has a deep understanding of natural language. There are two unsupervised pretraining tasks of BERT: the masking language model (MLM) and next sentence prediction (NSP) [18]. Learning on large-scale unlabeled training data through two pretraining tasks made BERT have great 
performance on various downstream nature language processing (NLP) tasks, such as machine translation, knowledge extraction, text classification and question answering systems [19-22].

Since there are few studies on designers' real reasoning thinking and sentence-level fuzzy semantic rezoning in product BID, this study conducts two experiments to determine designers' bionic reasoning thinking mode first, and then uses the natural language processing model BERT to realize bionic semantic reasoning.

\section{Experiment}

\subsection{Study 1: Bionic Reasoning Experiment from Product to Biology}

The purpose of this experiment is to explore designers' bionic reasoning modes and their frequency in bottom-up product bionic design.

\subsubsection{Subjects}

A total of 12 industrial design graduate students (7 male and 6 female, aged from 22 to 29), all of whom had more than four years of product design experience, were chosen as subjects to perform the bionic reasoning task.

\subsubsection{Stimuli}

Three experts discussed bionic products with the goal of covering as many product categories and product use environments as possible. Eight common products are selected: airplane, desk lamp, humidifier, motorcycle helmet, sofa, automobile, water cup, and speedboat, which cover complex products and simple products.

\subsubsection{Procedure}

Participants are invited to come up with as many organisms as possible according to each product provided in Section 2.1.2, assuming they are working on a product BID. After their reasoning, participants are asked to write down in brief sentences the reason for each product-to-biology combination.

The specific product appearance is not displayed to avoid interfering with the subjects' ideation. Meanwhile, subjects are given the authority to search for information from the internet.

\subsubsection{Results}

Statistics of the reasoning showed that on average, each subject inferred 3.09 corresponding organisms for each product. The common product-to-creature reasoning modes can be classified as 'affective perception of product- affective perception of creature reasoning', 'product form- creature form reasoning', 'product function- creature function reasoning', 'product using environment- creature habitat reasoning' and 'product materialcreature material reasoning'. When one reasoning mode refers to more than one creature, it is considered that the reasoning mode is used only once. Tables 1-5 show the main five reasoning modes, their frequency and examples. In addition, few subjects also used cultural association reasoning (four times), such as thinking of airplanes from tigers because of flying tigers.

Table 1. Affective perception of product-affective perception of creature reasoning (72 times).

\begin{tabular}{ccc}
\hline Product & Creature & Reason \\
\hline sofa & $\operatorname{dog}$ & $\begin{array}{c}\text { The big dog that is close to people can be lean } \\
\text { against, is big and gentle. }\end{array}$ \\
\hline helmet & hippopotamus head & $\begin{array}{c}\text { Hippopotamus head is very typical, honest and } \\
\text { heavy, with a sense of security. }\end{array}$ \\
\hline car & $\begin{array}{c}\text { cheetah, } \\
\text { tiger }\end{array}$ & $\begin{array}{c}\text { We can find some brave but fast animals to design } \\
\text { cars, such as cheetahs and tigers. }\end{array}$ \\
\hline
\end{tabular}


Table 2. Product form- creature form reasoning (49 times).

\begin{tabular}{ccc}
\hline Product & Creature & Reason \\
\hline airplane & eagle & $\begin{array}{c}\text { Eagles often fly in the sky in a soaring posture, } \\
\text { which looks more like an airplane in the air. }\end{array}$ \\
\hline desk lamp & turtle & $\begin{array}{r}\text { The shape of the tortoise shell is similar to the } \\
\text { countertop at the bottom of the lamp. }\end{array}$ \\
\hline cater cup & pelican & $\begin{array}{r}\text { The hump of a camel is like a container, and the beak } \\
\text { of a pelican is very big like a container. }\end{array}$ \\
\hline
\end{tabular}

Table 3. Product function- creature function reasoning (34 times).

\begin{tabular}{ccc}
\hline Product & Creature & Reason \\
\hline desk lamp & lantern fish & $\begin{array}{c}\text { From a functional point of view, first think of } \\
\text { animals that can emit light. The lure of the lantern } \\
\text { fish is luminous and has a 'fishing rod' structure, } \\
\text { which is more consistent with a desk lamp. }\end{array}$ \\
\hline speedboat & squid & Squids spray water backward and fast forward. \\
\hline humidifier & whale & Humidifier and whale both can spray water. \\
\hline
\end{tabular}

Table 4. Product using environment- creature habitat reasoning (20 times).

\begin{tabular}{ccc}
\hline Product & Creature & Reason \\
\hline speedboat & dolphin, shark & $\begin{array}{r}\text { Speedboats are in the water, I can directly think of } \\
\text { aquatic animals like dolphins and sharks. }\end{array}$ \\
\hline airplane & bald eagle, & Bald eagles live in the sky. \\
\hline
\end{tabular}

Table 5. Product material- creature material reasoning (19 times).

\begin{tabular}{ccc}
\hline Product & Creature & Reason \\
\hline $\begin{array}{c}\text { motorcycle } \\
\text { helmet }\end{array}$ & Snails, conch, pangolin & They all have very hard shell as motorcycle helmet. \\
\hline sofa & $\begin{array}{c}\text { rats, } \\
\text { brown bear, } \\
\text { tiger, orangutan, giraffe, } \\
\text { kiwi }\end{array}$ & $\begin{array}{c}\text { Sofa has the surface texture, which can imitate the } \\
\text { fur and patterns of wild animals, such as brown } \\
\text { bears, tigers, orangutans, and giraffes. } \\
\text { Kiwi, because it has a special hairy appearance. }\end{array}$ \\
\hline
\end{tabular}

The reasoning from a creature to a product can contain more than one reasoning mode. For example, an eagle is chosen as a creature with an aircraft as a target product, and the reason is "The movement method of eagle and aircraft both is flying and the shape of eagle wings is similar to wings of aircraft." It contains two reasoning modes: "product functionbiological function reasoning' and 'product form- biological form reasoning'.

\subsection{Study 2: Bionic Reasoning Experiment from Biology to Product}

\subsubsection{Subjects}

A total of 12 industrial design graduate students, as in Section 2.1.1, were chosen as subjects to perform the bionic reasoning task.

\subsubsection{Stimuli}

Three experts discussed organisms to infer from trying to cover different types of creatures. Eight types of creatures were chosen: bald eagles, cheetahs, polar bears, jellyfish, beetles, cacti, elephants, and bees.

According to the research of Luo and Dong [23], providing subjects with textual descriptions of cultural relics can obtain more innovative solutions than pictures. To avoid 
fixation in bionic reasoning, only the names of creatures are provided, and subjects are allowed to surf the internet.

\subsubsection{Procedure}

Based on eight biological samples, the participants were invited to deduce the related products of each creature as many times as possible through bionic reasoning and to describe the reason for each biology-to-product reasoning in short sentences.

\subsubsection{Results}

Statistics of the reasoning showed that, on average, each subject inferred 2.66 corresponding products for each creature. The creature-to-product reasoning modes can be classified as 'creature form- product form reasoning', 'affective perception of creature-affective perception of product reasoning', 'creature function- product function reasoning', 'creature material- product material reasoning', 'creature culture- product culture reasoning' and 'creature habitat reasoning- product using environment'. Tables 6-9 show the main reasoning modes, their frequencies and examples. In addition, culture associations (five times) and environmental connections (three times) also occurred in biology-to-product reasoning.

Table 6. Creature form- product form reasoning (60 times).

\begin{tabular}{ccc}
\hline Product & Creature & Reason \\
\hline cactus & toothpick holder & The thorns are pulled out like toothpicks. \\
\hline bald eagle & sickle & The outline is similar to the sickle. \\
\hline elephant & excavator & $\begin{array}{c}\text { The nose is shaped like an excavator. It is huge and } \\
\text { heavy, similar to an excavator. }\end{array}$ \\
\hline
\end{tabular}

Table 7. Affective perception of creature-affective perception of product reasoning (58 times).

\begin{tabular}{ccc}
\hline Product & Creature & Reason \\
\hline polar bear & warm products & $\begin{array}{c}\text { Polar bears look very warm and is suitable for } \\
\text { designing quilts, scarves and hats. }\end{array}$ \\
\hline bald eagle & high-end tea set & Bald eagle looks majestic and honorable. \\
\hline cheetah & motorcycle & Cheetah is agile, fast and energetic. \\
\hline
\end{tabular}

Table 8. Creature function- product function reasoning (27 times).

\begin{tabular}{ccc}
\hline Product & Creature & Reason \\
\hline beetle & climbing aid & $\begin{array}{c}\text { Beetles can climb on vertical branches or even glass } \\
\text { because of the barbs and suckers on their feet. }\end{array}$ \\
\hline bee & drone & Swarm collaboration, similar to drone fleet \\
\hline cactus & water reservoir & Cactus born in the desert but can save many water. \\
\hline
\end{tabular}

Table 9. Creature material- product material reasoning (26 times).

\begin{tabular}{ccc}
\hline Product & Creature & Reason \\
\hline cheetah & camouflage & $\begin{array}{c}\text { The cheetah's skin has leopard print, which is easy } \\
\text { to camouflage on the grassland. }\end{array}$ \\
\hline jellyfish & umbrella & $\begin{array}{c}\text { Jellyfish is transparent or transparent with color, it } \\
\text { looks like an umbrella. }\end{array}$ \\
\hline polar bear & sofa & Polar bear's cortex looks advanced. \\
\hline
\end{tabular}




\subsection{Discussion}

As shown in Table 10, on average, each subject could infer 3.09 matching organisms for each product. Each subject could infer 2.66 products for each organism with an average level. When inferring from the target domain product to the source domain organism, the subjects' thinking is more open. This phenomenon could be explained by early-stage uncertainties in factors such as the product's form, material, affective perception, and so on, causing the learner to pick less-educated guesses than it does under the normal circumstance. In a real design situation, the reasoning space may become smaller as more target users and functions of products are determined. In reverse inference from the source domain organism to the target domain product, participants' ideas are relatively limited. After the experiment, some subjects mentioned that it was more difficult to think from creatures than products. This may be explained by the fact that products are familiar to subjects, but creatures are far from participants' daily lives, which contributes to different knowledge backgrounds.

Table 10. Comparison of biology-to-product and product-to-biology reasoning models.

\begin{tabular}{ccc}
\hline Reasoning direction & Product to Biology & Biology to Product \\
\hline Output quantity & Biology Avg =3.09 & Product Avg = 2.66 \\
\hline & Affective Perception Reasoning (72) & Form Reasoning (60) \\
Reasoning mode and & Form Reasoning (49) & Affective Perception \\
frequency & Function Reasoning (34) & Reasoning (58) \\
& Environment Reasoning (20) & Function Reasoning (27) \\
& Material Reasoning (19) & Material Reasoning (26) \\
\hline Reasoning direction & Product to Biology & Biology to Product \\
\hline
\end{tabular}

The bionic mapping relationship between creatures and products is shown in Figure 2.

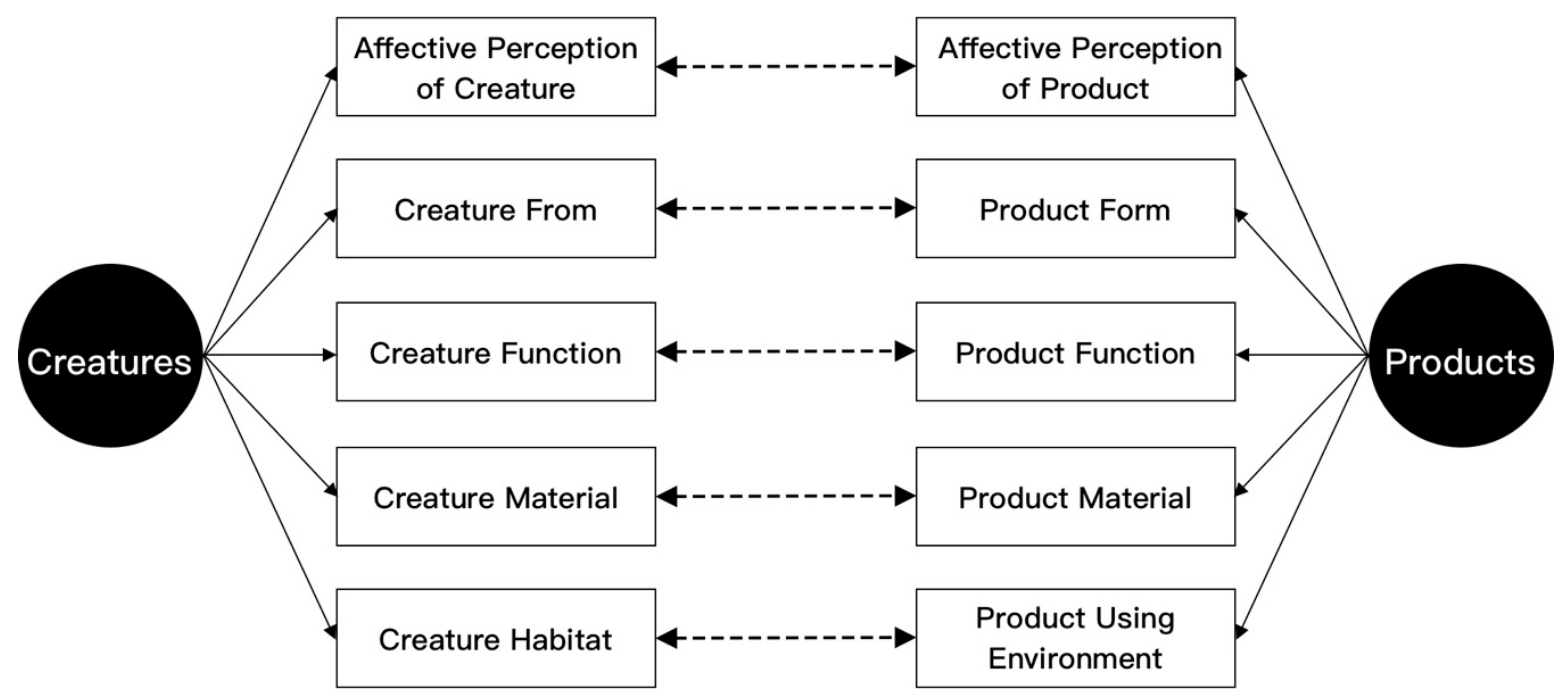

Figure 2. Bionic mapping relationship between creatures and products.

\section{Methodology}

Affective perception, function, material and habitat of creatures, as well as affective perception, function, material and using environment of products, can all be described in natural language. Meanwhile, biological form and product form can be better expressed through pictures.

This study introduces the bidirectional encoder representations from transformers (BERT) pretraining model to accomplish semantic reasoning of product BID. 


\subsection{Product and Biology Semantic Database}

A biological description dataset and product description dataset are established by describing the affective perception, function, environment, and material of common creatures and products. Some of the data are shown in Tables 11 and 12.

Table 11. Biological description dataset (display part of data).

\begin{tabular}{ccccc}
\hline Name & $\begin{array}{c}\text { Affective } \\
\text { Perception }\end{array}$ & Function & Habitat & Material \\
\hline giraffe & $\begin{array}{c}\text { docile; elegant; } \\
\text { beautiful; } \\
\text { friendly }\end{array}$ & $\begin{array}{c}\text { fight with the neck as a } \\
\text { weapon; } \\
\text { sleep in a standing position }\end{array}$ & $\begin{array}{c}\text { grassland, } \\
\text { shrubland, albizia } \\
\text { woodland }\end{array}$ & $\begin{array}{c}\text { short coat; } \\
\text { variegated } \\
\text { reticulation }\end{array}$ \\
\hline dolphin & $\begin{array}{c}\text { echolocation; } \\
\text { docile; friendly; } \\
\text { flexible }\end{array}$ & $\begin{array}{c}\text { good at jumping and } \\
\text { snorkeling; } \\
\text { swimming }\end{array}$ & ocean & $\begin{array}{c}\text { smooth and } \\
\text { hairless; } \\
\text { sharp legs }\end{array}$ \\
\hline owl & $\begin{array}{c}\text { nlert; } \\
\text { funny; } \\
\text { cute }\end{array}$ & $\begin{array}{c}\text { ntrong night vision; eyes } \\
\text { cannot turn in different } \\
\text { directions. }\end{array}$ & $\begin{array}{c}\text { tree; } \\
\text { rock; } \\
\text { grass; } \\
\text { sky }\end{array}$ & soft feathers \\
\hline
\end{tabular}

All the descriptions are written in Chinese.

Table 12. Product description dataset (display part of data).

\begin{tabular}{|c|c|c|c|c|}
\hline Name & $\begin{array}{l}\text { Affective } \\
\text { Perception }\end{array}$ & Function & $\begin{array}{c}\text { Using } \\
\text { Environment }\end{array}$ & Material \\
\hline $\operatorname{tank}$ & $\begin{array}{l}\text { large; } \\
\text { hidden }\end{array}$ & $\begin{array}{l}\text { reconnaissance; } \\
\text { firing artillery; } \\
\text { turret can rotate; } \\
\text { advance slowly }\end{array}$ & $\begin{array}{c}\text { jungle, } \\
\text { grassland, desert }\end{array}$ & sturdy \\
\hline ski board & fashion; cool & $\begin{array}{l}\text { reduce the pressure on } \\
\text { the gripping area on the } \\
\text { snow }\end{array}$ & snow field & firm; smooth \\
\hline $\begin{array}{l}\text { hot air } \\
\text { balloon }\end{array}$ & $\begin{array}{l}\text { enthusiasm; } \\
\text { lively }\end{array}$ & $\begin{array}{l}\text { flying; } \\
\text { the inside of the balloon } \\
\text { heats the air and uses } \\
\text { buoyancy to displace } \\
\text { the whole }\end{array}$ & in the air & $\begin{array}{l}\text { fire resistance; } \\
\text { good } \\
\text { airtightness; } \\
\text { bright colors }\end{array}$ \\
\hline
\end{tabular}

All the descriptions are written in Chinese.

\subsection{Product-Organism Semantic Similarity Calculation}

The process of calculating the semantic similarity of sentences based on BERT is shown in Figure 3.

(1) Input Sentence

Input a product description sentence and a biological description sentence.

(2) Word Embedding

The BERT model converts natural language into mathematical representation through word embedding. Each word in the input sentence is expressed as a word embedding with a fixed length of $\mathrm{m}$, which is convenient for subsequent processing and calculation.

(3) BERT Operation

After operation of the 12-layer transformer layer in the BERT model, the corresponding word embedding of the original sentence outputs. Suppose a sentence contains $n$ Chinese words; then, the word vector output of the sentence is denoted as $\overrightarrow{f_{1}}, \ldots \overrightarrow{f_{n}}$.

(4) Sentence embedding calculation 
Obtain the sentence embedding by calculating the mean value of the output word embedding. The sentence embedding is calculated as follows.

$$
\overrightarrow{\mathrm{f}}=\frac{\overrightarrow{\mathrm{f}_{1}}+\cdots+\overrightarrow{\mathrm{f}}_{\mathrm{n}}}{\mathrm{n}}
$$

(5) Cosine similarity calculation

Cosine similarity is used to calculate the similarity between two sentences. If the feature vector of sentence 1 is $\vec{A}$, the feature vector of sentence 2 is $\vec{B}$, the dimension of sentence embedding is $m$, and $A_{i}$ and $B_{i}$ are the components of $\vec{A}$ and $\vec{B}$, then the cosine similarity between the two sentences is calculated as follows.

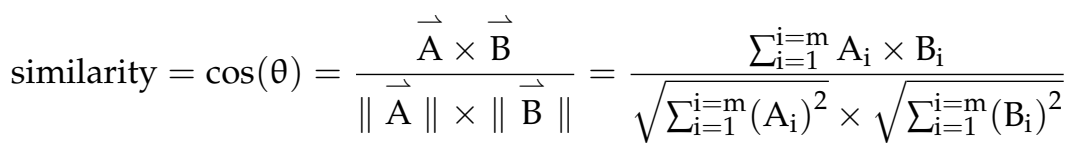

The value range of similarity is $[-1,1] .-1$ means the two vectors are opposite, and 1 means the two vectors are the same. The greater the cosine similarity value, the higher the semantic similarity of the two sentences.

Product Description Sentence1

(1)Input sentence

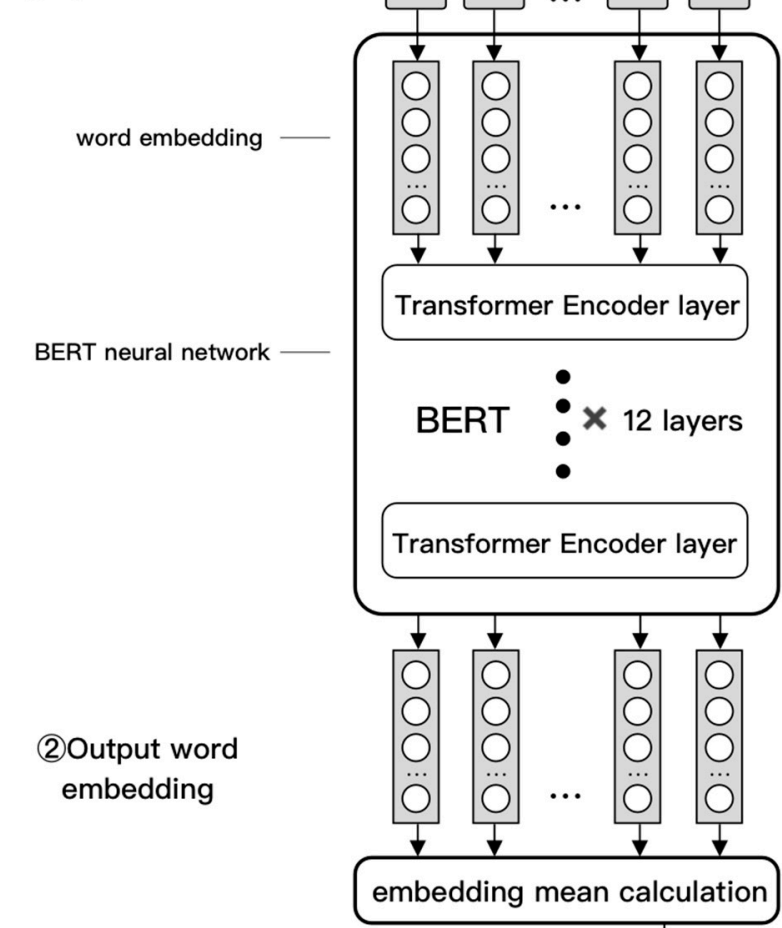

sentence embedding

(3) Semantic similarity calculation
Biological Description Sentence2

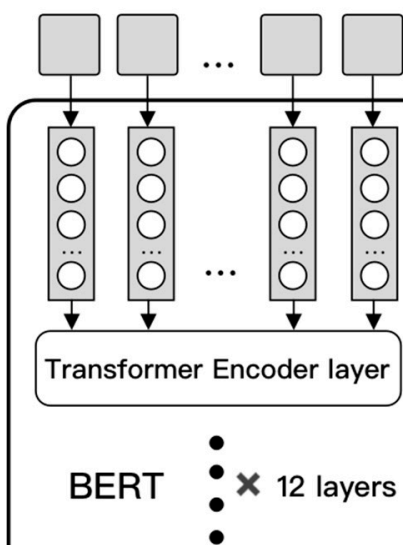

Transformer Encoder layer

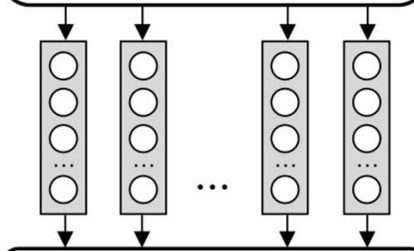

Embedding mean calculation

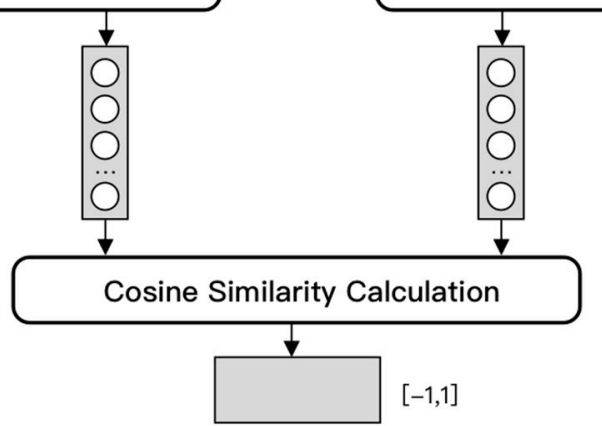

Figure 3. Product-organism semantic similarity calculation process based on the BERT pretraining model. 


\subsection{Semantic Reasoning of Product BID Based on BERT}

When inferring creatures through products, first input the description sentences of the product, and the semantic similarities between the product and every creature in the dataset of each reasoning mode are calculated using the algorithm in Section 3.2. Finally, the creatures inferred from each category are sorted according to the similarity from high to low (Figure 4). Designers can choose creatures with high similarity as the source to start bionic expressions. It is similar to infer from creature to product.

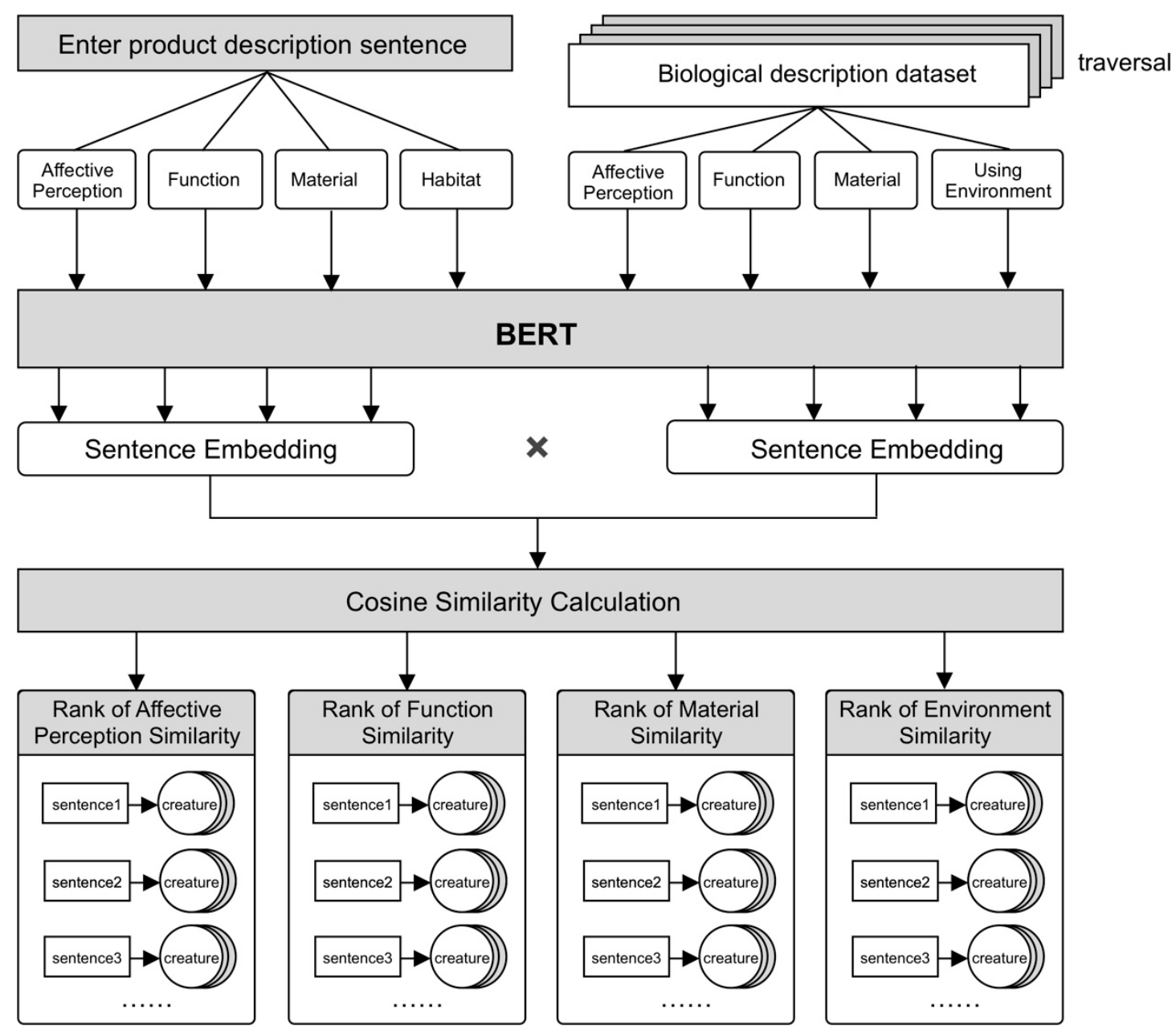

Figure 4. Semantic reasoning process from product to biology.

\subsection{Example}

A bionic semantic reasoning system is developed on RoBERTa-wwm-ext, a derived Chinese model of BERT-base, which adapts whole word masking in the masked language model (MLM) pretraining task $[24,25]$. RoBERTa has been applied to text classification in many fields [26-29].

When using night light as the target product to search for suitable creatures for product BID, the designer first inputs descriptions of the night light, as shown in Table 13. The semantic reasoning results are shown in Figure 5 and Tables 14-17. 
Table 13. Description of night light.

\begin{tabular}{cc}
\hline Affective Perception & fashion; warm \\
\hline Function & illuminate \\
\hline Material & light \\
\hline Using Environment & in the room \\
\hline All the terms are written in Chinese. &
\end{tabular}

All the terms are written in Chinese.
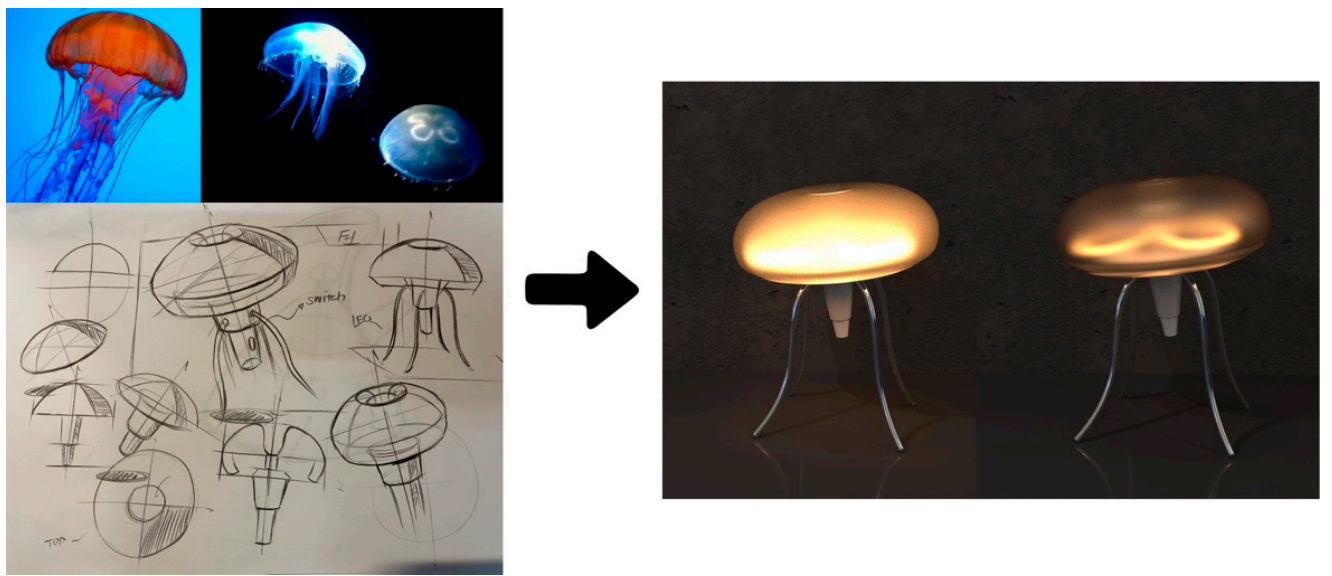

Figure 5. Bionic night light design reference, concept sketch and rendering effect.

Table 14. Semantic reasoning result of affective perception.

\begin{tabular}{ll}
\hline Inference sentence: fashion \\
\hline $\begin{array}{l}\text { Result } \\
\text { ['fashion', [Flamingo]), ('handsome', [bald eagle, zebra, cheetah]), ('light', } \\
\text { crane, swan, puppet cat]), ('sexy', [hippo]) }\end{array}$ \\
\hline Similarity & [1.0000000, 0.7545206, 0.7223246, 0.7198027, 0.7121808] \\
\hline Inference sentence: warm & ('warm', [polar bear, giant panda, sunflower]), ('homesick', [emperor penguin]), \\
\hline Result & $\begin{array}{l}\text { ('docile', [African elephant, giraffe, dolphin, lop-eared rabbit, sheep, pig, puppet } \\
\text { cat]), ('friendly', [pigeon, golden retriever, puppet cat]), ('lazy', [puppet cat]) }\end{array}$ \\
\hline Similarity & [0.8465299, 0.7907890, 0.7823601, 0.7615751, 0.7563425] \\
\hline All the terms are displayed in Chinese.
\end{tabular}

Table 15. Semantic reasoning result of function.

\begin{tabular}{ll}
\hline \multicolumn{2}{l}{ Inference sentence: illuminate } \\
\hline Result & ('glow', [jellyfish]), ('strong night vision', [owl]), ('phototaxis', [praying mantis]), \\
('active at night', [bat]), ('night walk', [owl, tiger])
\end{tabular}

All the terms are displayed in Chinese.

Table 16. Semantic reasoning result of material.

\begin{tabular}{ll}
\hline Inference sentence: light \\
\hline \multirow{2}{*}{ Result } & $\begin{array}{l}\text { ('light', [jellyfish]), ('supple', [golden retriever]), ('soft', [puppet cat]), ('smooth', } \\
\text { [orca, beetle, ant, bamboo']), ('fluffy', [ostrich]) }\end{array}$ \\
\hline Similarity & [1.0000000, 0.8214510, 0.7871054, 0.7731293, 0.7605499] \\
\hline
\end{tabular}

All the terms are displayed in Chinese. 
Table 17. Semantic reasoning result of using environment.

\begin{tabular}{|c|c|}
\hline \multicolumn{2}{|c|}{ Inference sentence: in the room } \\
\hline Result & $\begin{array}{l}\text { ('in the air', [bee, red-crowned crane]), ('sky', [bald eagle, owl, flamingo, } \\
\text { woodpecker, pigeon]), ('on the tree', [woodpecker]), ('desert', [camel, cactus]), } \\
\text { ('ocean', [jellyfish, killer whale, dolphin]) }\end{array}$ \\
\hline Similarity & {$[0.7273592,0.6848752,0.6745558,0.6739956,0.6695887]$} \\
\hline
\end{tabular}

According to the inference result, jellyfish ranked first in function reasoning, and the function description "illuminate" of night light corresponds to "glow" of jellyfish's function. At the same time, jellyfish also ranked in the top five in environmental reasoning and affective perception reasoning. Therefore, jellyfish was chosen as the source domain creature of the night light.

The designer searched for more jellyfish pictures for reference, drew the conceptual sketches, performed modeling and rendering in the 3D modeling software Rhinoceros, and finally completed the product shape bionic design of the jellyfish night light (Figure 5).

\section{Discussion and Conclusions}

This study first conducted two experiments to determine designers' bionic reasoning thinking in top-down and bottom-up processes and obtained bionic mapping relationships, including affective perception, form, function, material and environment. Based on the reasoning modes, the NLP pretraining model BERT was used to assist the bionic semantic reasoning. Sentence-level fuzzy semantic reasoning of product-to-creature and creature-toproduct can both be realized.

The advantages of this method are listed as follows.

(1) Through experimental research, designers' bionic reasoning thinking modes such as affective perception, form, function, material, and environment are obtained. Comparing with the previous bionic reasoning, which lacks research on real reasoning thinking, the applied methodology simulates designers' real ideation in product BID, which makes bionic semantic reasoning more comprehensive and scientific.

(2) Unlike the keyword-based precise reasoning in the past, the usage of BERT in bionic reasoning helps achieve sentence-level fuzzy search in BID for the first time. Moreover, comparing with perception matching, the applied methodology does not require much labor on manual scoring, which improves the search range and efficiency of bionic reasoning.

(3) During the study, the researchers have built two scalable datasets, including a biological description dataset and a product description dataset, and both can serve as a foundation to broaden designers' bionic reasoning thinking and knowledge, which will be helpful for product BID education and industry.

Future works of this article are listed as follows.

(1) First, work is the improvement of the datasets. Data in the biological dataset and product dataset are limited; therefore, the final reasoning similarity is not extremely high. Meanwhile, this study uses manual methods to get data instead of crawling because the data required are relatively special. In the future, we will expand the dataset and look for ways to automate the sourcing process.

(2) At present, the reasoning results under each reasoning mode are displayed independent of one another. One creature can be recommended by two different modes at the same time. Thus, a comprehensive recommendation system based on weights assigned to different modes is a potential future project to develop. At the same time, morphological reasoning and topological reasoning mentioned in Section 1.2 can also be included.

(3) At last, this semantic reasoning method can be applied in other analogy design fields, such as cultural product design, which takes cultural artefact as the source domain and product as the target domain. 
Author Contributions: Z.B. designed and performed the experiments, analyzed the experimental data, and wrote the manuscript. S.L. designed the experiments and gave advice on experiment design and manuscript writing. F.Z. programed and realized bionic semantic reasoning function by BERT. L.W. and P.S. provided the datasets and drew the figures. All authors have read and agreed to the published version of the manuscript.

Funding: This study was supported by the National Natural Science Foundation of China (No. 52075478), the key project of National Social Science Foundation of China (No. 21AZD056), the major art project of National Social Science Foundation of China (No. 20ZD09-5), and the major project of Zhejiang Social Science Foundation (No. 21XXJC01ZD).

Institutional Review Board Statement: Not applicable.

Informed Consent Statement: Not applicable.

Data Availability Statement: Not applicable.

Conflicts of Interest: The authors declare no conflict of interest.

\section{References}

1. Alipour, L.; Faizi, M.; Moradi, A.M.; Gholamreza, A. The impact of designers' goals on design-by-analogy. Des. Stud. 2017, 51, 1-24. [CrossRef]

2. Nachtigall, W.; Wisser, A. Building and climatization. In Bionics by Examples, 1st ed.; Springer: Cham, Switzerland, 2015; pp. 117-133.

3. Aziz, M.S. Biomimicry as an approach for bio-inspired structure with the aid of computation. Alex. Eng. J. 2016, 55, 707-714. [CrossRef]

4. Neurohr, R.; Dragomirescu, C. Bionics in engineering-defining new goals in engineering education at "politehnica" university of bucharest. Conf. Eng. Educ. 2007, 1, 3-6. Available online: http://icee2007.dei.uc.pt/proceedings/papers/571.pdf (accessed on 13 December 2021).

5. Helms, M.; Vattam, S.S.; Goel, A.K. Biologically inspired design: Process and products. Des. Stud. 2009, 30, 606-622. [CrossRef]

6. Chai, C.; Cen, F.; Ruan, W.; Yang, C.; Li, H. Behavioral analysis of analogical reasoning in design: Differences among designers with different expertise levels. Des. Stud. 2015, 36, 3-30. [CrossRef]

7. Luo, S.; Bian, Z.; Hu, Y. How can biological shapes inspire design activity in closed domains? Int. J. Technol. Des. Educ. 2020, 1-27. Available online: https:/ /link.springer.com/article/10.1007/s10798-020-09593-y (accessed on 13 December 2021). [CrossRef]

8. Cheong, H.; Shu, L.H. Using templates and mapping strategies to support analogical transfer in biomimetic design. Des. Stud. 2013, 34, 706-728. [CrossRef]

9. Mak, T.W.; Shu, L.H. Using descriptions of biological phenomena for idea generation. Res. Eng. Des. 2008, 19, 21-28. Available online: https: / link.springer.com/article/10.1007\%2Fs00163-007-0041-y (accessed on 13 December 2021). [CrossRef]

10. Shu, L.H.; Cheong, H. A Natural Language Approach to Biomimetic Design. In Biologically Inspired Design, 1st ed.; Goel, A.K., McAdams, D.A., Stone, R.B., Eds.; Springer: Cham, Switzerland, 2015; pp. 29-61. [CrossRef]

11. Chiu, I.; Shu, L.H. Bridging cross-domain terminology for biomimetic design. In Proceedings of the International Design Engineering Technical Conferences and Computers and Information in Engineering Conference, Long Beach, CA, USA, 24-28 September 2005; ASME: New York, NY, USA, 2008; Volume 4742, pp. 93-101. [CrossRef]

12. Chiu, I.; Shu, L.H. Biomimetic design through natural language analysis to facilitate cross-domain information retrieval. $A I$ EDAM 2007, 21, 45-59. [CrossRef]

13. Cheong, H.; Shu, L.H.; Stone, R.B.; McAdams, D.A. Translating terms of the functional basis into biologically meaningful keywords. In Proceedings of the International Design Engineering Technical Conferences and Computers and Information in Engineering Conference, New York, NY, USA, 3-6 August 2008; ASME: New York, NY, USA, 2009; Volume 43284, pp. 137-148. [CrossRef]

14. Luo, S.; Zhang, Y.; Zhang, J.; Xu, J.H. A User Biology Preference Prediction Model Based on the Perceptual Evaluations of Designers for Biologically Inspired Design. Symmetry 2020, 12, 1860. [CrossRef]

15. Yuan, X.Q.; Chen, D.K.; Ynag, Y.P.; Qi, B. Bionic imagery associated with product form design. Comput. Eng. Appl. 2014, 50, 178-182. [CrossRef]

16. Luo, S.J.; Bian, Z.; Zhang, Y.F.; Lu, J.W.; Lu, S.Z. A study on product bionic fusion design based on shape matching. Comput. Integr. Manuf. Syst. 2020, 26, 2633-2641. [CrossRef]

17. Zhu, H. Cognitive Coupling Based Product Image Morphology Bionic Evolutionary Design Method. Master's Thesis, Lanzhou University of Technology, Lanzhou, China, 2018.

18. Devlin, J.; Chang, M.W.; Lee, K.; Toutanova, K. Bert: Pre-training of deep bidirectional transformers for language understanding. In Proceedings of the Conference of the North American Chapter of the Association for Computational Linguistics: Human Language Technologies, NAACL HLT, Minneapolis, MN, USA, 2-7 June 2019; Volume 1, pp. 4171-4186. 
19. Zhang, Z.; Wu, S.; Jiang, D.W.; Chen, G. BERT-JAM: Maximizing the utilization of BERT for neural machine translation. Neurocomputing 2021, 460, 84-94. [CrossRef]

20. Harnoune, A.; Rhanoui, M.; Mikram, M.; Yosifi, S.; Elkaimbillah, Z.; Asri, B.E. BERT Based Clinical Knowledge Extraction for Biomedical Knowledge Graph Construction and Analysis. Comput. Methods Programs Biomed. Update 2021, 1, 100042. [CrossRef]

21. Shah, S.M.A.; Taju, S.W.; Ho, Q.T.; Nguyen, T.T.D.; Qu, Y.Y. GT-Finder: Classify the family of glucose transporters with pre-trained BERT language models. Comput. Biol. Med. 2021, 131, 104259. [CrossRef] [PubMed]

22. Wu, Z.; Liang, J.; Zhang, Z.; Lei, J. Exploration of text matching methods in Chinese disease Q\&A systems: A method using ensemble based on BERT and boosted tree models. J. Biomed. Inform. 2021, 115, 103683. [CrossRef] [PubMed]

23. Luo, S.J.; Dong, Y.N. Role of cultural inspiration with different types in cultural product design activities. Int. J. Technol. Des. Educ. 2017, 27, 499-515. [CrossRef]

24. Cui, Y.; Che, W.; Liu, T.; Qin, B.; Yang, Z. Pre-training with whole word masking for Chinese BERT. In Proceedings of the IEEE/ACM Transactions on Audio, Speech, and Language Processing, Maynooth, Ireland, 28 July-1 August 2015; IEEE: Piscataway, NJ, USA, 2021. [CrossRef]

25. GitHub. Available online: https://github.com/ymcui/Chinese-BERT-wwm (accessed on 25 August 2021).

26. Liu, Y.; Liu, H.; Wong, L.P.; Lee, L.K.; Zhang, H.; Hao, T. A Hybrid Neural Network RBERT-C Based on Pre-trained RoBERTa and $\mathrm{CNN}$ for User Intent Classification. In Proceedings of the International Conference on Neural Computing for Advanced Applications, Shenzhen, China, 27-30 August 2020; Zhang, H., Zhang, Z., Wu, Z., Hao, T., Eds.; Springer: Cham, Switzerland, 2020; pp. 306-319. [CrossRef]

27. Liao, W.; Zeng, B.; Yin, X.; Wei, P. An improved aspect-category sentiment analysis model for text sentiment analysis based on RoBERTa. Appl. Intell. 2021, 51, 3522-3533. [CrossRef]

28. Maldonado-Sifuentes, C.E.; Angel, J.; Sidorov, G.; Kolesnikova, O.; Gelbukh, A. Virality Prediction for News Tweets Using RoBERTa. In Proceedings of the Advances in Soft Computing, MICAI, Mexico City, Mexico, 25-30 October 2021; Batyrshin, I., Gelbukh, A., Sidorov, G., Eds.; Springer: Cham, Switzerland, 2021; pp. 88-95. [CrossRef]

29. Wang, X.; Iwaihara, M. Integrating RoBERTa Fine-Tuning and User Writing Styles for Authorship Attribution of Short Texts. In Proceedings of the Asia-Pacific Web (APWeb) and Web-Age Information Management (WAIM) Joint International Conference on Web and Big Data, Guangzhou, China, 23-25 August 2021; U, L.H., Spaniol, M., Sakurai, Y., Chen, J., Eds.; Springer: Cham, Switzerland, 2021; pp. 413-421. [CrossRef] 